\title{
Expansão da Área Agrícola e Produtividade das Culturas no Brasil: testando hipóteses da legislação californiana de biocombustíveis ${ }^{1}$
}

\author{
Elaine Correa Daubermann², Lucas Squarize Chagas ${ }^{3}$, \\ Angelo Costa Gurgel $^{4}$ e Sérgio Naruhiko Sakurai ${ }^{5}$
}

Resumo: O presente estudo investiga a hipótese de que a conversão de áreas de florestas e pastagens em áreas agrícolas no Brasil reduz a produtividade das mesmas em 50\%. Essa hipótese vem sendo utilizada pelo Air Resource Board da Califórnia para estimar as emissões de gases de efeito estufa associados a mudanças indiretas no uso da terra provenientes da expansão da produção de biocombustíveis. Com base em testes econométricos, os resultados apresentados pela maioria dos modelos sugerem não ocorrer queda da produtividade quando da expansão da área de culturas no País, ou, quando ocorrem, são pouco expressivas, sinalizando, portanto, a necessidade de revisão e correção dos parâmetros da política californiana de combustíveis de baixas emissões.

Palavras-chaves: Produtividade agrícola, mudanças no uso da terra, emissões de gases de efeito estufa, padrão de combustíveis de baixas emissões.

Abstract: We investigate the hypothesis that the conversion of forest and pasture land to cropland in Brazil decreases yields by 50\%. Such hypothesis is being used by the California Air Resource Board to estimate the greenhouse gas emissions from indirect land use changes due to the expansion of biofuels production. We formulate several econometric models to test such hypothesis. The results from the majority of the models indicate that yields do not decrease under the expansion of cropland area in the country, or the decrease

1. Os autores agradecem os comentários de dois pareceristas anônimos desta revista, eximindo-os de quaisquer erros remanescentes. Agradecem também o suporte financeiro provido pelo CNPq e pela Fapesp.

2. Graduada em Economia - FEA-RP/USP. E-mail: elainecdaubermann@yahoo.com.br

3. Graduado em Economia - FEA-RP/USP e Mestrando em Economia - IPE-FEA/USP. E-mail: lsquarize@gmail.com

4. Docente da Escola de Economia de São Paulo da Fundação Getúlio Vargas - EESP/FGV. E-mail: angelo.gurgel@fgv.br

5. Docente do Departamento de Economia - FEA-RP/USP. E-mail: sakurai@usp.br 
in yields is very small. Therefore, our results indicate the need for revising and correcting the parameters in the Californian low carbon emissions policy.

Key-words: Agricultural yield, land use changes, greenhouse gas emissions, low carbon fuel standard.

Classificação JEL: Q24, Q15, Q54.

\section{Introdução}

O mundo vive o dilema do desenvolvimento sustentável, devendo ponderar os efeitos do crescimento econômico sobre o meio ambiente. É consensual a necessidade de redução de emissão de gases de efeito estufa para que não ocorram mudanças consideráveis no clima do planeta nos próximos anos. O Painel Intergovernamental sobre Mudanças Climáticas (IPCC), responsável por avaliar e sintetizar os principais trabalhos científicos sobre mudanças climáticas, afirma em relatório que as temperaturas médias na superfície do globo terrestre devem elevar-se provavelmente entre $1,1^{\circ}$ e $6,4^{\circ} \mathrm{C}$ entre 1990 e 2100 (IPCC, 2007).

Ademais, há grande preocupação com a matriz energética atual, altamente dependente do finito petróleo. Em face disso, diversas iniciativas têm sido tomadas para expandir o uso de biocombustíveis no mundo, buscando aliar solução à questão ambiental e ao problema de escassez de petróleo. Porém, essa alternativa abre espaço para outra preocupação: a de que as áreas de produção de biocombustíveis venham competir com as áreas florestais e de produção de alimentos.

Dentre as preocupações associadas à expansão dos biocombustíveis, destacam-se os possíveis impactos negativos advindos de mudanças no uso da terra. Acredita-se que o aumento na demanda e na produção de biocombustíveis pode estar relacionado à redução na área plantada de culturas agrícolas tradicionais nos países desenvolvidos e aumento na pressão para expansão da fronteira agrícola pelo desmatamento e conversão de áreas nativas em países em desenvolvimento.

Considerando-se apenas a emissão de gás carbônico $\left(\mathrm{CO}_{2}\right)$ na combustão de biocombustíveis e a captura deste gás da atmosfera durante seu crescimento vegetal, os biocombustíveis são incontestavelmente fontes limpas de energia, que ajudariam a enfrentar o problema do efeito estufa. Porém, quando se consideram as emissões de gases de efeito estufa advindas dos combustíveis e fertilizantes utilizados na sua produção, bem como as possíveis emissões decorrentes de mudanças diretas e indiretas no uso da terra pela expansão das áreas de produção de biomassa para biocombustível, os benefícios ambientais proporcionados pelos mesmos passam a ser questionados.

Searchinger et al. (2008), por exemplo, afirmam que os estudos que apontaram saldo positivo líquido de sequestro de $\mathrm{CO}_{2}$ pelos biocombustíveis não consideraram as emissões advindas de mudança no uso da terra. Os autores utilizaram um modelo mundial que projeta aumentos em terras agrícolas por país em resposta a um possí- 
vel aumento na produção de etanol de milho dos EUA de 56 bilhões de litros acima dos níveis projetados para 2016. Concluíram que haveria o dobro de emissões de gases de efeitos estufa ao longo de 30 anos e saldo positivo de emissões por 167 anos com o etanol de milho. Para o etanol celulósico, o aumento destas emissões seria menor, mas ainda assim seriam necessários 52 anos para que o saldo líquido fosse favorável à redução em emissões.

Outro estudo que investiga como as mudanças no uso da terra podem limitar o potencial de redução de emissões dos biocombustíveis foi conduzido por Fargione et al. (2008). Esses autores estimaram o tempo necessário para que fossem pagos os débitos de carbono provocados pela expansão da produção de biocombustíveis sobre diferentes ecossistemas. Para isso, consideraram os débitos de carbono liberado da biomassa dos ecossistemas e os impactos de mudança no uso da terra baseados em outros estudos científicos. Foram analisados os impactos do biodiesel de soja na Amazônia do Brasil, do biodiesel de soja no Cerrado brasileiro, do etanol de cana-de-açúcar no Cerrado brasileiro, do biodiesel de palma em floresta tropical da Indonésia ou Malásia, do biodiesel de palma em turfa de floresta tropical da Indonésia ou Malásia e do etanol de milho em regiões de grama na parte central dos Estados Unidos. Os resultados mostraram que os tempos de payback ${ }^{6}$ do aumento de gases estufa são, respectivamente, iguais a 320,37, 17, 86, 840 e 93 anos, para cada ecossistema.

Melillo et al. (2009), por sua vez, consideram a contabilidade dinâmica completa das emissões de mudanças no uso da terra, considerando a expansão de biocombustíveis avançados como consequência de uma política global de redução de emissões de gases de efeito estufa. Esses autores concluem que os biocombustíveis geram emissões consideravelmente inferiores à gaso-

6. Tempo necessário para que um dado tipo de biocombustível passe a gerar emissões líquidas negativas quando são consideradas as emissões provocadas pela conversão do ecossistema original em plantação de biomassa e a redução em emissões que o biocombustível proporciona em relação ao combustível fóssil que o mesmo é capaz de substituir. lina, benefício que aumenta quanto maior for o período de produção dos biocombustíveis.

Neste contexto, o álcool produzido da cana-de-açúcar é um promissor biocombustível em termos ambientais, além de apresentar alta produtividade por área e custo competitivo. Contudo, as condições climáticas e hídricas requeridas agem como limitadoras da ampliação de sua produção mundial. Por um lado, tem predominado no cenário internacional uma desconfiança de que a expansão deste biocombustível pode levar a mudanças de uso da terra nos principais países fornecedores, como o Brasil. Por outro lado, argumenta-se também que o Brasil e outros países tropicais ainda possuem considerável disponibilidade de terra, o que resultaria em pequeno efeito de substituição de áreas florestais por áreas produtoras de biocombustíveis.

Diversas iniciativas estão sendo desenvolvidas em diferentes países com o intuito de estimular o aumento do uso de biocombustíveis e a substituição de combustíveis fósseis. Nos EUA, o Energy Policy Act of $2005^{7}$ visa aumentar a produção de biocombustíveis, principalmente o etanol, determinando a mistura do mesmo com a gasolina. De acordo com este mandato, o nível de produção de etanol para mistura deveria atingir os 6,1 bilhões de galões em 2009 (25\% de aumento em relação à produção em 2006) e 7,5 bilhões em 2012 (53\% de aumento), sendo principalmente visada a produção de etanol de milho. Na União Europeia (UE), o European Union Biofuels Directive requer que os biocombustíveis representem pelo menos $10 \%$ dos mercados de combustíveis líquidos em 2020 (EUROPEAN COMMISION, 2007). Neste caso, o biodiesel é o principal combustível sendo estimulado.

De particular interesse para o presente estudo é a iniciativa de padrões de combustíveis de baixas emissões (Low Carbon Fuel Standard) do estado da Califórnia, proposta de regulação que visa impor a redução gradual nas emissões dos combustíveis consumidos naquele estado, publicada em março de 2009 pela California Environmental

7. http://www.epa.gov/oust/fedlaws/publ_109-058.pdf 
Protection Agency (CALIFORNIA AIR RESOURCE BOARD, 2009). O relatório desta regulação será denominado relatório CARB (California Air Resource Board) no presente estudo.

O relatório CARB visa a adoção de um padrão de combustível de baixo carbono no setor de transportes californiano, via redução gradual da intensidade de carbono (IC) dos combustíveis, de 95,61 gramas de $\mathrm{CO}_{2}$ equivalente por Mega Joule $\left(\mathrm{gCO}_{2}-\mathrm{e} / \mathrm{MJ}\right)$, valor base para a gasolina, para 86,27 $\mathrm{gCO}_{2}$-e/MJ, entre 2011 e 2020. Entre os demais objetivos, consta reduzir a dependência de petróleo pela Califórnia, criar um mercado de tecnologia de transporte limpo e estimular a produção de combustíveis de baixo carbono.

Para mensurar a IC de diferentes tipos de combustíveis, o relatório CARB fez uso da análise de ciclo de vida das emissões de gases de efeito estufa, que contempla os efeitos diretos e indiretos. Os primeiros são associados à produção, transporte e uso, como por exemplo, o tipo de fertilizante aplicado na produção de biomassa e sua frequência, a coleta e transporte da safra e o combustível utilizado no processo de produção, entre outros. Os efeitos indiretos são associados à mudança de uso da terra ocasionada pela produção de biocombustíveis, mensurados por meio do modelo econômico de equilíbrio geral Global Trade Analysis Project (GTAP) (Hertel, 1997). A versão do modelo GTAP utilizada neste caso procura mensurar como uma mudança na produção de um bem agrícola em um país afeta o uso da terra em todo o mundo, considerando as relações entre os diferentes setores e mercados e o comércio internacional.

Segundo o relatório CARB, a intensidade de carbono associada aos efeitos diretos da produção de etanol de cana-de-açúcar, calculada pelos modelos de ciclo de vida, varia de 12,4 $\mathrm{g} \mathrm{CO}_{2}$-e/ $\mathrm{MJ}$ a 27,4 $\mathrm{gCO}_{2}$-e/MJ, enquanto o efeito indireto de mudança de uso da terra, estimado a partir do modelo GTAP, é de $46,0 \mathrm{gCO}_{2}$-e/ $\mathrm{MJ}^{8}$, quase o dobro do máximo de emissões diretas. Isso implica em uma intensidade de carbono total de até 73,4

8. http://www.arb.ca.gov/fuels/lcfs/121409lcfs_lutables.pdf
$\mathrm{gCO}_{2}$-e/MJ, o que indica que este biocombustível poderia ser utilizado para satisfazer a proposta de regulação, dependendo das proporções em que seja misturado com a gasolina, apesar de este valor ser consideravelmente alto em relação a outros exercícios de mensuração da intensidade de emissões do etanol da cana-de-açúcar9.

As emissões decorrentes de mudanças indiretas de uso da terra no relatório $\mathrm{CARB}$ foram tomadas como emissões médias em cinco cenários alternativos de simulação do modelo GTAP, que diferem de acordo com valores de parâmetros comportamentais do modelo, permitindo considerar incertezas sobre o fenômeno sendo investigado. Um dos parâmetros importantes na construção do modelo é a razão assumida entre a produtividade de novas áreas de culturas, convertidas a partir de pastagens e florestas ${ }^{10}$, e a produtividade de áreas tradicionais na produção de culturas. Esse parâmetro determina, para cada região e zona agroecológica (AEZ) ${ }^{11}$ representada no GTAP, qual seria a redução na produtividade de uma nova área de cultura, convertida a partir de áreas de pastagens ou de áreas de vegetação natural, em relação à produtividade das áreas de cultuas já existentes, quando houver a necessidade de expansão da área agrícola em alguma região. O modelo assume, para os choques de expansão do etanol de cana-de-açúcar, que essa razão de produtividade da área nova em relação às tradicionais é de 0,50 em três cenários e 0,75 em um cenário, para todos os países e regiões do mundo. Ainda, um último cenário assume o valor de 0,50 para todos os países, exceto o Brasil, que recebe o valor de 0,80 para este parâmetro.

Os valores utilizados pelo modelo GTAP para o parâmetro de razão da produtividade entre áreas novas e áreas tradicionais foram determinados com base na percepção de especialistas de

9. Ver, por exemplo, Melillo et al. (2009) e EPA (2010).

10. As áreas de florestas no modelo GTAP incluem, em parte, as áreas de Cerrado com maior composição arbórea.

11. Zonas geográficas que apresentam características similares de solo, clima e precipitação, determinando características relativamente homogêneas de aptidão agrícola e potencial de cultivo. 
países desenvolvidos. A hipótese básica considera que a decisão racional dos agricultores é de preferir o uso das áreas agrícolas de maior aptidão, e que estas já se encontram em uso no mundo. Desta forma, futuras expansões da produção de culturas devem ocorrer em áreas de menor produtividade, em geral, $50 \%$ inferior do que das áreas tradicionais de cultivo. Contudo, esta hipótese não foi testada empiricamente e ignora a grande disponibilidade de áreas subutilizadas para agricultura em algumas regiões tropicais do mundo, como é o caso da América Latina e, particularmente, do Brasil. Posto de outra forma, ainda, não foi utilizada nenhuma metodologia quantitativa para estimar os valores deste parâmetro utilizados no GTAP.

Vale ressaltar que diversos estudos destacam os ganhos de produtividade como um importante elemento da expansão da produção brasileira nas últimas décadas. Gasques et al. (2010), por exemplo, mencionam o aumento no rendimento por área de diversas lavouras ao longo do tempo, como uva, café, milho, soja, trigo e cana-de-açúcar, e estimam que os incrementos na produtividade total dos fatores (PTF) da agropecuária brasileira foram da ordem de 2,13\% ao ano no período de 1995 a 2006. De acordo com Albuquerque e Silva (2008), o aumento da produção por área nas últimas décadas é consequência das melhorias tecnológicas proporcionadas principalmente pelos investimentos em pesquisa. $\mathrm{Na}$ mesma direção, Alves et al. (2013) destacam que a produtividade da terra, influenciada pela tecnologia, foi o componente dominante na explicação do crescimento da produção agrícola brasileira. Matha Jr. et al. (2012), por sua vez, apontam que a expansão da pecuária brasileira de 1985 em diante se deu com o aumento da produtividade dos rebanhos e queda da área total utilizada por esta atividade. Não foram encontrados, contudo, na literatura, estudos que tenham estimado as mudanças na produção por área quando da expansão da fronteira agrícola. Considerando-se a falta de evidência empírica e de estudos quantitativos sobre o parâmetro de razão de produtividade, o objetivo do presente estudo consiste em investigar a validade da hipótese de redução na produtividade de áreas agrícolas novas quando da expansão da área total de culturas no Brasil. Espera-se, com isso, verificar se os resultados de coeficientes de intensidade de carbono para o etanol da cana-de-açúcar na proposta de legislação californiana se sustentam diante de valores mais realistas para este parâmetro comportamental do modelo, contribuindo, assim, para a discussão sobre a capacidade dos biocombustíveis em reduzir as emissões de gases de efeito estufa.

\section{Metodologia}

O objetivo do trabalho é testar a hipótese de que a expansão das áreas de culturas no Brasil está associada à redução da sua produtividade. Para tal, foram formulados e estimados modelos econométricos que testam como a mudança na área de culturas (variável explicativa) está relacionada à produtividade (variável dependente). Este teste representa uma análise do parâmetro utilizado no modelo GTAP, que mede a mudança na produtividade pela expansão de área, definido no Relatório CARB como elasticity of crop yields with respect to area expansion, que consiste na razão entre a produtividade de terra recém-transformada em agrícola ("área novas") e a produtividade da terra tradicionalmente agrícola

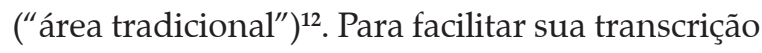
neste estudo, este parâmetro será denominado razão de produtividades entre área nova e área tradicional.

Foram utilizadas duas abordagens de análise da relação entre produtividade e área plantada, chamados aqui de Modelo A e Modelo B. O primeiro baseia-se no estudo de Bruce e Carriquiry (2010) sobre o mesmo tema, com alterações na construção da variável dependente e no uso de uma base de dados comparativamente mais ampla. O segundo modelo consiste em regredir

12. Vale ressaltar que, apesar de ser chamado de elasticidade pelo relatório CARB, esse parâmetro não atende ao conceito de elasticidade utilizado em economia, tratando-se apenas de uma razão entre duas variáveis. 
a variação percentual anual do índice de produtividade agrícola agregado em relação à variação percentual anual da área plantada. Ressalta-se, também, que cada modelo A e B de regressão foi estimado por meio de duas especificações: na primeira, o índice de produtividade foi regredido apenas em função da variação da área plantada; a segunda, por sua vez, envolve a inclusão de uma variável dummy, denominada $\psi$, que assume o valor um para variação de área positiva (ou nula) e o valor zero para variação de área negativa. Enquanto a primeira especificação permite analisar a influência das variações (positivas e negativas) da área plantada sobre a produtividade, a segunda especificação permite captar se há diferenças na relação entre a variável dependente e independente quando a área se expande ou decresce, separadamente. Dados os propósitos do artigo, este procedimento procura aprofundar a análise da relação entre área plantada e produtividade da terra no Brasil. A seguir, são apresentadas as equações que representam os modelos A e B, assim como uma discussão mais específica sobre cada um destes modelos.

\subsection{Modelo A}

Bruce e Carriquiry (2010) buscaram analisar a razão de produtividades entre área nova e área tradicional, formulando um modelo algébrico aplicado à cultura da soja no Brasil. $\mathrm{O}$ modelo considera o período $t$ como o ano-base, que define que as terras existentes até então são terras tradicionais $\left(A_{::}^{\text {old }}\right)$, e as terras incorporadas à agricultura $\left(A_{t+n}^{\text {new }}\right)$ a partir de então são consideradas terras novas. Os autores definem a produtividade da terra $\bar{Y}: \because$ no período $t+n$ como:

$$
\bar{Y}_{t+n}^{::}=\frac{A^{\text {old }} Y_{t+n}^{\text {old }}+A_{t+n}^{\text {new }} Y_{t+n}^{\text {new }}}{A^{\text {old }}+A_{t+n}^{\text {new }}}
$$

em que $A_{t}^{\text {new }}=0, Y_{t+n}^{\text {old }}$ é a produtividade da terra tradicional e $Y_{t+n}^{\text {new }}$ é a produtividade da terra nova convertida ao uso agrícola.

Chamando a razão de produtividades entre área nova e área tradicional de $\gamma$ e supondo
$Y_{t+n}^{\text {new }}=\gamma Y_{t+n}^{\text {old }}$, a diferença entre a produtividade da terra no período $t+n$ e do período $t$ torna-se:

$$
\begin{aligned}
& \bar{Y}_{t+n}-\bar{Y}_{t}=\frac{A^{\text {old }}}{A^{\text {old }}+A_{t+n}^{\text {new }}}\left(Y_{t+n}^{\text {old }}-Y_{t}^{\text {old }}\right)+ \\
& +\frac{A_{t+n}^{\text {new }}}{A^{\text {old }}+A_{t+n}^{\text {new }}}\left(\gamma Y_{t+n}^{\text {old }}-Y_{t}^{\text {old }}\right)
\end{aligned}
$$

o que é uma média ponderada do crescimento de produtividade na área tradicional e do crescimento de produtividade da terra nova, ajustado por suas respectivas participações na área total.

Estabelecendo que $\alpha=\frac{A^{\text {old }}}{A^{\text {old }}+A_{t+n}^{\text {new }}}$, pode-se reescrever a equação (2) como:

$\bar{Y}_{t+n}^{:}-\bar{Y}_{t}=\alpha_{n}\left(Y_{t+n}^{\text {old }}-Y_{t}^{\text {old }}\right)+\left(1-\alpha_{n}\right)\left(\gamma Y_{t+n}^{\text {old }}-Y_{t}^{\text {old }}\right)$

Supondo que o crescimento da produtividade das áreas tradicionais é constante ao longo do período considerado, tem-se $\delta=Y_{t+1}^{\text {old }}-Y_{t}^{\text {old }}$. A partir disso, pode-se derivar a equação (4) abaixo:

$$
\begin{aligned}
& \bar{Y}_{t+n}-\bar{Y}_{t}=\alpha_{n} n \delta+\left(1-\alpha_{n}\right) \gamma Y_{t}^{\text {old }}+ \\
& +\left(1-\alpha_{n}\right) n \gamma \delta-\left(1-\alpha_{n}\right) Y_{t}^{\text {old }}
\end{aligned}
$$

Embora não seja possível realizar uma regressão com a formulação em (4), uma vez que $\left(1-\alpha_{n}\right)$ é correlacionado com $\alpha_{n}$, caso não haja diferença de produtividade entre as áreas tradicionais e novas, isto é, se $\gamma=1$, então, a partir da equação (4) tem-se:

$$
\frac{\bar{Y}_{t+n}-\bar{Y}_{t}}{n}=\delta
$$

A partir da equação (5) obtém-se a expressão que será estimada econometricamente:

$$
\frac{\bar{Y}_{t+n}-\bar{Y}_{t}}{n}=\beta_{0}+\beta_{1} \cdot X_{n}
$$

Em que $X_{n}$ é a variável que corresponde à participação da terra nova sobre a terra total, isto é, $\frac{A_{t+n}^{\text {new }}}{A^{\text {old }}+A_{t+n}^{\text {new }}}$.

Com base no anteriormente exposto, testar a hipótese de que $\gamma=1$ equivale a testar a hipótese de que $\beta_{l}=0$ (hipótese nula), de que a expansão da área não afeta a produtividade, contra a 
hipótese alternativa $\beta_{l} \neq 0$, de que um aumento na área de culturas está associado a alterações na produtividade média.

Bruce e Carriquiry (2010) estimaram a expressão (6) para o período de 1996 a 2010 usando os dados de produtividade da soja, cultura no Brasil predominantemente associada às regióes de fronteiras agrícolas. Os autores utilizaram uma divisão do Brasil em seis regiões: Norte (Amazônia), Mapito $^{13}$ e Bahia, Nordeste Litorâneo, Sudeste e Sul, adicionando cinco dummies de intercepto para captar diferenças regionais de variação de produtividade. Dois tipos de regressão foram considerados, quais sejam, uma em que a variável explicativa $X_{n}$ representa a participação da nova área agrícola para todas as culturas, e outra para a participação da nova área agrícola somente ocupada com soja.

Os autores encontraram coeficientes negativos, mas estatisticamente não significantes, levando à conclusão de que o valor da razão de produtividades entre área nova e área tradicional mais razoável de se supor seria um, ou seja, que não há diferença de produtividade da terra nova em relação à terra tradicional. Os autores argumentam ainda que esta razão de produtividades entre área nova e área tradicional é razoável para um país de extensões como as do Brasil, em que custos de transporte limitam a produção mais do que a própria produtividade intrínseca da terra.

Contudo, o uso da produtividade da cultura da soja pode enviesar a análise, uma vez que essa cultura está entre as de maior nível tecnológico e emprego de insumos modernos. Uma alternativa seria, então, considerar a produtividade média das principais culturas brasileiras, por meio de um índice de produtividade.

Contudo, para a regressão com o índice de produtividade agrícola brasileiro, a interpretação do coeficiente estimado deve ser modificada por alterar a dimensão da variável dependente, que passa de diferença em nível entre as produtividades de cada ano (quando a produtividade era a da cultura da soja apenas) para diferença

13. Mapito: Maranhão, Piauí e Tocantins. em pontos percentuais entre a variação de produtividade (mensuradas por meio do índice) de cada ano. Desta forma, esta nova especificação deve ser modificada em sua derivação em relação ao modelo original de Bruce e Carriquiry (2010). $\mathrm{O}$ índice de produtividade das culturas pode ser estabelecido como na equação (7):

$$
I_{k}^{t}=\alpha_{i k} \times \frac{P_{i k}^{t}}{P_{i k}^{t_{0}}}+\alpha_{j k} \times \frac{P_{j k}^{t}}{P_{j k}^{t o}}+\ldots+\alpha_{s k} \times \frac{P_{s k}^{t}}{P_{s k}^{t o}}
$$

Em que o índice de produtividade I do estado $k$ no ano $t$ (entre 1996 e 2008) em relação ao ano $t_{0}$ (qual seja, o ano de 1995) é obtido via cálculo da média ponderada das produtividades das culturas em cada estado em relação ao ano $t_{0}$. Na equação (7), $i, j$ e $s$ definem as diferentes culturas, $P$ é a produtividade da cultura $i$ no estado $k$ e ano $t$, $\alpha_{i k}$ é o fator de ponderação da cultura $i$ no estado $k$, sendo $\alpha_{i k}=\frac{V P_{k}^{i}}{V P_{k}}$, sendo VP o valor da produção da cultura $i$ no estado $k$.

A diferença entre $I_{k}^{t}$ e $I_{k}^{t_{0}}$ fornece as variações percentuais de produtividade média em relação ao ano-base $t_{0}$, ponderadas por $\alpha_{i k}$ para cada estado, conforme equação (8):

$$
\begin{aligned}
& I_{k}^{t}-I_{k}^{t_{0}}=\alpha_{i k} \times\left(\frac{P_{i k}^{t}}{P_{i k}^{t_{0}}}-\frac{P_{i k}^{t_{0}}}{P_{i k}^{t_{0}}}\right)+\alpha_{j k} \times\left(\frac{P_{j k}^{t}}{P_{j k}^{t 0}}-\frac{P_{j k}^{t_{j}}}{P_{j k}^{t_{j}}}\right)+ \\
& +\ldots+\alpha_{s k} \times\left(\frac{P_{s k}^{t}}{P_{s k}^{t_{0}}}-\frac{P_{s k}^{t_{0}}}{P_{s k}^{t_{0}}}\right)
\end{aligned}
$$

A equação (8) pode ser reescrita como:

$$
\begin{aligned}
& I_{k}^{t}-I_{k}^{t_{0}}=\alpha_{i k} \times\left(\frac{P_{i k}^{t}}{P_{i k}^{t_{i}}}-1\right)+\alpha_{j k} \times\left(\frac{P_{j k}^{t}}{P_{j k}^{t_{0}}}-1\right)+ \\
& +\ldots+\alpha_{s k} \times\left(\frac{P_{s k}^{t}}{P_{s k}^{t_{0}}}-1\right)
\end{aligned}
$$

Assim, a variação em pontos percentuais anuais linearizados da produtividade de cada cultura do ano corrente em relação ao ano-base, para cada unidade da federação k, é descrita como $\frac{I_{k}^{t}-I_{k}^{t_{0}}}{t-t_{0}}$. Portanto, as duas regressões estimadas para o índice de produtividade agrícola do Brasil no Modelo A são definidas como: 


$$
\begin{gathered}
\frac{I_{k}^{t}-I_{k}^{t_{0}}}{t-t_{0}}=\beta_{0}+\beta_{l} \cdot X_{n}+\varepsilon_{k}^{t_{0} t_{0}} \\
\frac{I_{k}^{t}-I_{k}^{t_{0}}}{t-t_{0}}=\beta_{0}+\beta_{2} \cdot \psi \cdot X_{n}+\beta_{3} \cdot(1-\psi) \cdot X_{n}+\varepsilon_{k}^{t^{t_{0}}}
\end{gathered}
$$

Em que, conforme anteriormente mencionado, $\psi$ é uma dummy igual a um quando $X_{n}$ é maior ou igual a zero e igual a zero quando $X_{n}$ é menor que zero e $\varepsilon_{k}^{t t_{0}}$ se refere ao erro da estimação. Desta forma, enquanto o parâmetro $\beta_{1}$ mensura o efeito médio das variações da área cultivada sobre a produtividade, a significância estatística dos parâmetros $\beta_{2}$ e $\beta_{3}$ define se a produtividade varia de forma assimétrica frente a expansões e quedas da área plantada, respectivamente.

Foram estimadas regressões com o índice de produtividade agregado para todos os estados, perfazendo um total de 351 observações. Desta forma, o Modelo A pretende tornar mais abrangente o modelo proposto por Bruce e Carriquiry (2010), pela presença de $\psi$ e pela maior amplitude de informações quando utilizado com o índice de produtividade agrícola brasileiro e não com apenas uma única cultura representativa. Ainda, aqueles autores consideram apenas seis regiões representando o país, enquanto o presente estudo considera cada unidade da federação como uma observação. Estas alterações podem, portanto, ser vistas como contribuições adicionais deste trabalho.

\subsection{Modelo B}

O Modelo B refere-se à regressão em que a variável dependente consiste na variação percentual anual da produtividade e a variável independente é a variação percentual anual da área plantada, para o mesmo intervalo de tempo. Essa formulação procura evitar a fixação do ano-base em um ponto relativamente distante no passado, como é o caso do Modelo A.

Com o índice de produtividade agrícola para o Brasil, a variável dependente também representa a variação percentual anual, mas em função da formulação do índice, sua construção foi direta, obtida a partir da construção do próprio índice, conforme demonstrado a seguir:

$$
\begin{aligned}
& \frac{\gamma_{k}^{t}-\gamma_{k}^{t-1}}{\gamma_{k}^{t-1}}=\alpha_{i k} \times\left(\frac{P_{i k}^{t}}{P_{i k}^{t-1}}-1\right)+ \\
& +\alpha_{j k} \times\left(\frac{P_{j k}^{t}}{P_{j k}^{t-1}}-1\right)+\ldots+\alpha_{s k} \times\left(\frac{P_{s k}^{t}}{P_{s k}^{t-1}}-1\right)
\end{aligned}
$$

Em que $\gamma_{k}^{t}$ consiste na produtividade agrícola do estado $k$ no ano $t$ em relação à produtividade agrícola do estado $\mathrm{k}$ no ano $t_{0}$, ponderado por $\alpha_{i k}$.

Neste caso, como a variação percentual refere-se ao ano imediatamente anterior, e não ao ano-base como no Modelo A, a produtividade de cada ano foi dividida pela produtividade do ano anterior para cada cultura em cada estado. Assim, a variável dependente, $\frac{\gamma_{k}^{t}-\gamma_{k}^{t-1}}{\gamma_{k}^{t-1}}$, representa a variação percentual anual das produtividades de cada cultura multiplicadas pelo fator ponderador de participação no valor de produção, para cada estado. As duas equações do Modelo B referentes ao índice de produtividade podem ser representadas como:

$$
\begin{aligned}
& \frac{\gamma_{k}^{t}-\gamma_{k}^{t-1}}{\gamma_{k}^{t-1}}=\delta_{0}+\delta_{1} \cdot \frac{A_{t}-A_{t-1}}{A_{t-1}}+\varepsilon_{k}^{t, t-1} \\
& \frac{\gamma_{k}^{t}-\gamma_{k}^{t-1}}{\gamma_{k}^{t-1}}=\delta_{0}+\delta_{2} \cdot \psi \cdot \frac{A_{t}-A_{t-1}}{A_{t-1}}+ \\
& +\delta_{3} \cdot(1-\psi) \cdot\left(\frac{A_{t}-A_{t-1}}{A_{t-1}}\right)+\varepsilon_{k}^{t, t-1}
\end{aligned}
$$

Logo, para o índice de produtividade agrícola como variável dependente, no Modelo A, a variável dependente exprime os pontos percentuais anuais linearizados do referido índice, enquanto no Modelo B, a variável dependente representa a variação percentual anual do índice de produtividade agrícola para o Brasil. Assim como no modelo A, a significância estatística dos parâmetros $\delta_{2}$ e $\delta_{3}$ provê indícios de que aumentos e quedas da área cultivada afetam de forma assimétrica a produtividade, respectivamente.

Os dois modelos diferem quanto ao conceito de área nova, pois, no Modelo A, a variação de área plantada é considerada como a variação de área ocorrida entre o ano em questão e o ano-base, 
ou seja, considerando toda a variação de área acumulada no período até então. Já no Modelo B, a variação de área plantada é expressa como a variação de área do ano em questão em relação ao ano imediatamente anterior, isto é, é uma variação percentual anual, como se o tempo para uma área ser considerada área nova fosse menor. Em termos comparativos, pode-se considerar que o Modelo A estabelece uma relação de médio e longo prazo entre variação de produtividade e de área plantada, enquanto o Modelo B estabelece uma relação de curto prazo (variações entre anos consecutivos) - trata-se, portanto, de visões complementares sobre o problema investigado.

Alguns esclarecimentos adicionais merecem ser apresentados. Em primeiro lugar, dado que tanto o Modelo A quanto o Modelo B trabalham com diferenças no tempo, as diferenças de produtividade inerentes a cada unidade da federação (ou seja, os efeitos fixos de cada estado) são automaticamente retirados das equações de interesse. Isto equivale a dizer que as estimações já levam em conta os (ou já controlam pelos) efeitos idiossincráticos de cada estado quanto à produtividade agrícola. Outra ressalva importante diz respeito ao fato de que, em períodos curtos (um ano), as variações em produtividade agrícola devem-se quase que exclusivamente a variações climáticas (ROBERTS e SCHLENKER, 2010; HUANG e KHANNA, 2010), o que tenderia a mascarar os efeitos que a expansão das áreas de culturas teria sobre a produtividade em função da ocupação de áreas de menor aptidão agrícola. Portanto, apesar de o Modelo B evitar a limitação do Modelo A de "ancorar" os resultados à produtividade e área do ano-base, o Modelo B pode não captar a relação entre mudança na área e produtividade por considerar, no termo de erro, variações climáticas, que tendem a ser bem mais expressivas que a relação sendo investigada. Desta forma, visando controlar pelos efeitos específicos de cada ano da amostra, a exemplo dos fatores climáticos, tanto as regressões do Modelo A como do Modelo B incluem dummies de ano.

Uma última consideração importante é o reconhecimento de que os vários aspectos sob o controle do agricultor capazes de alterar a produtividade da atividade agrícola, como investimentos em melhores técnicas de manejo e uso de insumos modernos (correção do solo e adubação de culturas, uso de sementes de cultivares modernos e mais adaptados e emprego de defensivos agrícolas, entre outros), não foram considerados na análise econométrica, uma vez que o objetivo é apenas testar a hipótese do relatório CARB de que novas áreas agrícolas possuem, em média, produtividade equivalente à metade daquela observada em áreas tradicionais. Desta forma, a necessidade de expandir a agricultura em áreas de menor potencial para cultivo, seja por menor aptidão agrícola, limitações em qualidades físicas e químicas do solo, bem como condições de temperatura e precipitação menos favoráveis, poderia ser acompanhada de maiores gastos com tecnologia e manejo, que compensariam a queda em produtividade. Contudo, os custos maiores desta expansão precisariam ser compensados por preços mais favoráveis das commodities agrícolas, sendo mais racional para o agricultor buscar primeiro a expansão sobre áreas com maiores aptidões. Desta forma, os modelos econométricos aqui formulados não são capazes de captar a possibilidade de maiores investimentos compensarem quedas de produtividade na expansão das áreas de culturas na agricultura brasileira, o que pode ser interpretado como uma hipótese implícita de que a expansão sobre novas áreas é feita mantendo-se o nível tecnológico médio adotado nas áreas tradicionais, ou acompanhando o ritmo de progresso tecnológico observados nessas áreas ${ }^{14}$.

14. Pode-se argumentar que os aumentos em preços dos produtos agrícolas, estimulados pela crescente demanda por alimentos advinda do crescimento populacional e da renda, promovem incentivos tanto para a intensificação da produção nas áreas tradicionais de cultivos quanto para a expansão da fronteira agrícola. A expansão sobre áreas de aptidão agrícola semelhante à das áreas tradicionais permitiria a sustentação da produtividade da terra sem maiores custos, enquanto a expansão sobre áreas de menor aptidão agrícola deveria ser acompanhada de redução na produtividade (hipótese do relatório CARB) e/ ou aumentos nos custos de produção. 


\subsection{Base de dados para a análise econométrica}

Para se estimar a relação entre a variação da área cultivada e a produtividade agregada das culturas, foi considerado um painel de 27 estados brasileiros durante os anos de 1995 a 2008. Os resultados foram obtidos por via do procedimento OLS clássico com desvios padrão robustos a heterocedasticidade e autocorrelação de resíduos (método Huber-White-Sandwich) ${ }^{15}$.

Discutindo de modo mais específico as variáveis utilizadas, como a razão de produtividade entre área nova e área tradicional se refere à expansão de área sobre terras não utilizadas para culturas anteriormente (como pastagens e florestas), considerou-se na coleta de dados a área plantada agregada de todas as culturas, temporárias e permanentes, no Brasil. Assim, a expansão da área plantada só pode ocorrer sobre áreas não utilizadas anteriormente para culturas ${ }^{16}$.

Os dados de produtividade estão disponíveis apenas por cultura, não havendo um único valor de produtividade para o total de culturas no Brasil. Torna-se necessário, então, estimar um índice de produtividade para todas as culturas. Desta forma, buscou-se construir um índice de produtividade agrícola para os estados brasileiros que represente as culturas mais importantes para o País e que também permita a agregação de forma consistente das culturas com diferentes padrões de produtividade.

Os dados sobre área e produção foram coletados da Produção Agrícola Municipal (PAM), no site do Instituto Brasileiro de Geografia e Estatística (IBGE). As culturas selecionadas para construção do índice de produtividade foram: algodão herbáceo, arroz, aveia, batata-inglesa,

15. Estimações efetuadas no programa Stata 9.2.

16. A consideração da variação de área de apenas uma ou algumas culturas específicas poderia mascarar a possibilidade de que uma mudança na área não ocorre necessariamente sobre uma área nova ou não utilizada para cultivo (áreas de florestas e pastagens), uma vez que essa cultura poderia estar substituindo alguma outra cultura, casos em que a área já era de uso para cultivo, e, portanto, não serviria ao objetivo da análise. cana-de-açúcar, centeio, cevada, feijão, fumo, girassol, mandioca, milho, soja, sorgo, tomate, trigo, triticale, algodão arbóreo, banana, borracha, cacau, café, laranja, limão, maçã, manga, tangerina e uva. Essas culturas contemplam de $86,44 \%$ a $95,48 \%$ do valor de produção total de todas as culturas no Brasil no período considerado (1995 a 2008). Assim, foram obtidos os valores da variável rendimento médio (razão entre a quantidade produzida ${ }^{17}$ e a área colhida ${ }^{18}$ ) na mesma escala das outras culturas, que apresentam seus rendimentos médios em $\mathrm{kg} / \mathrm{ha}$ na base de dados do IBGE.

O ano-base escolhido neste estudo foi 1995. Como a grandeza da produtividade varia entre culturas, o valor da produtividade de cada cultura para cada ano foi dividido pelo valor da produtividade da mesma em 1995 ou no ano anterior, de forma a obter-se a mesma grandeza para todas as variáveis. Nos casos em que não havia produtividade em alguns anos, optou-se por inserir a produtividade média do período analisado antes do cálculo do índice agregado anual por estado.

$O$ índice de produtividade agregado da agricultura foi calculado para cada estado ponderando-se a razão das produtividades acima descrita pela participação do valor de produção de cada cultura no valor de produção total de seus respectivos estados. Essas participações variam no tempo, e essa variação temporal poderia distorcer o índice agrícola agregado de forma a mascarar o real comportamento da produtividade em si, sendo influenciado pelo efeito de substituição de culturas de produtividades diferentes no estado sobre a produtividade geral do estado. Então, optou-se por calcular essas ponderações considerando o valor de produção médio no período considerado, mantendo-se as ponderações fixas para todos os anos, o que significa que a participação atribuída a cada cultura

17. Quantidade total colhida de cada produto agrícola no município, durante o ano de referência da pesquisa (IBGE).

18. Total de área efetivamente colhida de cada produto agrícola no município, durante o ano de referência da pesquisa (IBGE). 
Figura 1. Variação em pontos percentuais do índice de produtividade agrícola brasileiro por ano versus participação da área nova sobre a área plantada total no Brasil de 1995 a 2008 (Modelo A)

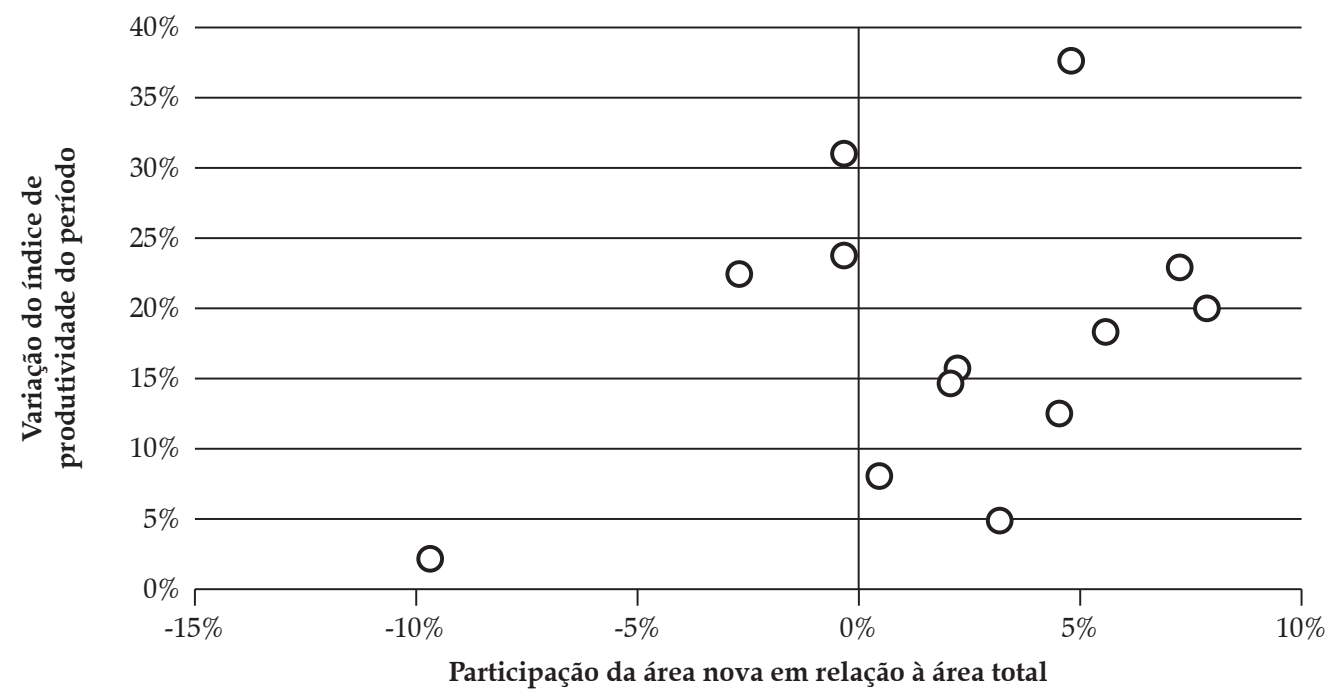

Fonte: Elaborado pelos autores a partir dos dados da pesquisa.

no valor de produção geral do estado representa a participação média no período analisado. Essa formulação também ameniza o efeito da variação brusca de preços em alguns anos específicos, que poderia afetar o valor de produção e modificar o índice de produtividade agregado. Os valores de produção foram corrigidos monetariamente pelo Índice de Preços por atacado, IPA-EP'19 (matérias-primas brutas agropecuárias), da Fundação Getúlio Vargas (FGV).

As razões de produtividade foram multiplicadas pelas ponderações, de forma a se alcançar um índice de produtividade ponderado por cultura. A soma de cada parcela correspondente a cada cultura por estado resultou no índice de produtividade agrícola agregado para cada estado.

\section{Resultados e discussão}

Esta seção apresenta os resultados das estimações realizadas com o objetivo de avaliar a relação entre expansão da área de culturas e variação na produtividade agrícola no Brasil, por meio das

19. Índice de Preços por Atacado segundo Estágios de Processamento, obtido do banco de dados do IPEA (www. ipeadata.gov.br) formulações dos modelos A e B expostos anteriormente. Apresentam-se, inicialmente, algumas estatísticas sobre os dados utilizados nas regressões e a seguir, os resultados das estimações.

A Figura 1 ilustra o comportamento da variável dependente versus a variável independente, construídas conforme o Modelo A, para valores agregados ao nível nacional. Cada ponto na figura representa uma observação anual. A figura sugere uma relação positiva, apesar de bastante dispersa, entre as variáveis.

A Figura 2 ilustra o comportamento da variável dependente versus a variável independente conforme o Modelo B para valores agregados para o Brasil. Observa-se uma relação fraca entre as duas variáveis que, como discutido anteriormente, pode estar relacionada ao forte efeito das variações climáticas anuais sobre a produtividade.

A Tabela $1^{20}$ apresenta o resultado das regressões de valores dos parâmetros estimados (com sua respectiva estatística $t$ entre parênteses).

20. Na Tabela 1, bem como nas demais tabelas de resultados, optou-se por não apresentar os coeficientes e estatísticas t das dummies de ano, por economia de espaço e para facilitar a visualização dos resultados principais. Conforme discutido na metodologia, essas dummies visam captar as diferenças em produtividades decorrentes de efeitos específicos de cada ano da amostra. 
Figura 2. Variação percentual anual do índice de produtividade versus variação percentual anual da área plantada total no Brasil de 1995 a 2008 (Modelo B)

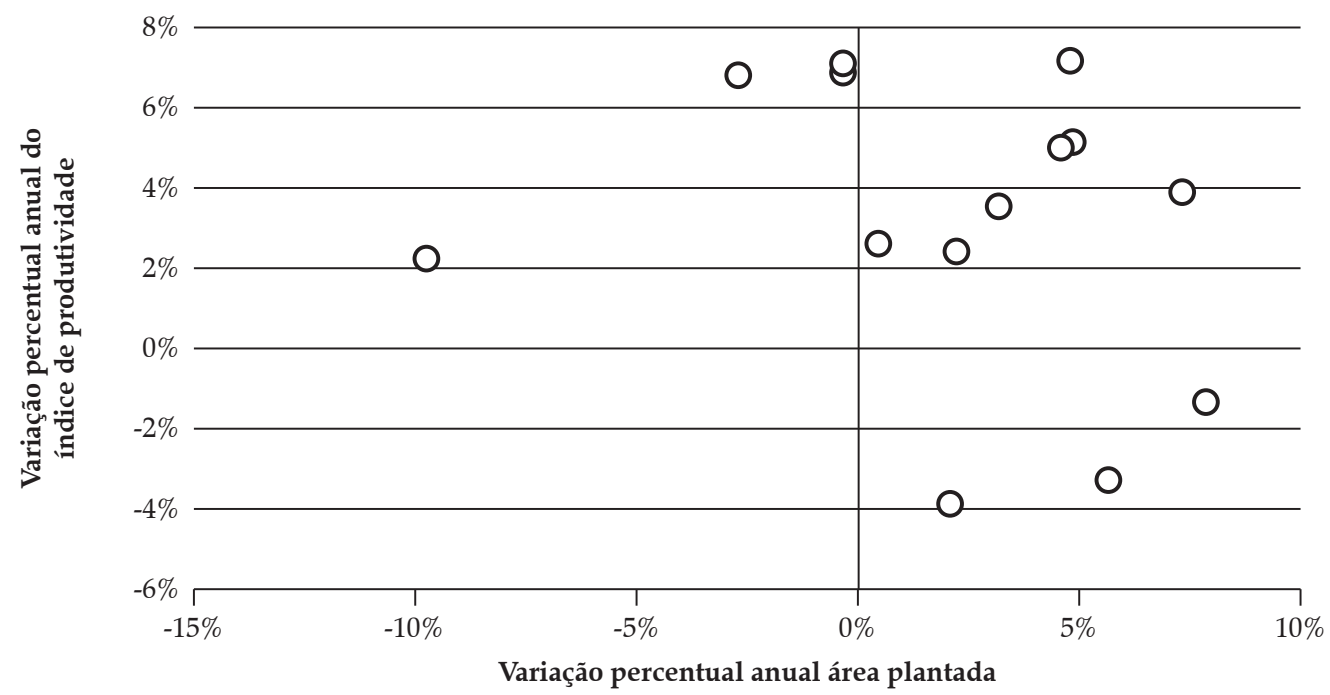

Fonte: Elaborado pelos autores a partir dos dados da pesquisa.

Tabela 1. Resultados das regressões: Variação de índice de produtividade agregado em relação à variação de área plantada do Brasil de 1995 a 2008

\begin{tabular}{|c|c|c|c|c|}
\hline \multirow{2}{*}{ Coeficiente (Estatística t) } & \multicolumn{2}{|c|}{ Modelo B } & \multicolumn{2}{|c|}{ Modelo A } \\
\hline & (1) & (2) & (3) & (4) \\
\hline Variação da área $\left(\beta_{1}\right.$ ou $\left.\delta_{1}\right)$ & $\begin{array}{c}0,085 \\
(1,150) \\
\end{array}$ & & $\begin{array}{c}0,156^{* * *} \\
(4,768) \\
\end{array}$ & \\
\hline Variação positiva da área $\left(\beta_{2}\right.$ ou $\left.\delta_{2}\right)$ & & $\begin{array}{c}-0,018 \\
(-0,205) \\
\end{array}$ & & $\begin{array}{c}-0,033 \\
(-0,501) \\
\end{array}$ \\
\hline Variação negativa da área $\left(\beta_{3}\right.$ ou $\left.\delta_{3}\right)$ & & $\begin{array}{c}0,280^{* *} \\
(2,575) \\
\end{array}$ & & $\begin{array}{c}0,311^{* * *} \\
(6,622)\end{array}$ \\
\hline Constante & $\begin{array}{c}-0,022 \\
(-0,664) \\
\end{array}$ & $\begin{array}{c}0,014 \\
(0,367) \\
\end{array}$ & $\begin{array}{c}0,001 \\
(0,020) \\
\end{array}$ & $\begin{array}{c}0,043 \\
(1,232) \\
\end{array}$ \\
\hline $\mathrm{R}^{2}$ & 0,108 & 0,113 & 0,232 & 0,264 \\
\hline Observações (N x T) & 351 & 351 & 351 & 351 \\
\hline
\end{tabular}

Notas: Estatística $t$ entre parênteses; *** Significativo a 1\%; ** Significativo a 5\%; * Significativo a 10\%. Dummies de ano incluídas nas estimações. Fonte: Resultados da pesquisa.

Os resultados apresentados nas colunas 1 e 3, referentes à especificação que não distingue variações positivas das variações negativas da área cultivada, indicam uma relação positiva entre variação da produtividade e variação da área, embora o coeficiente seja significativo somente na especificação referente ao Modelo A. Neste caso, de acordo com a coluna (3), os resultados indicam que a cada elevação de um ponto percentual na variação anual da área, o índice de produtividade varia em 0,15 ponto percentual positivo. Contudo, conforme as colunas 2 e 4, em que os efeitos de elevações e reduções de área são tratados separadamente, nota-se que os parâmetros referentes a aumentos da área cultivada são sempre estatisticamente nulos, implicando, assim, que às tais expansões não estão associadas quedas na produtividade agrícola - desta forma, tem-se um resultado bastante diferente do assumido pelo relatório CARB que, conforme discutido anteriormente, assume que as novas áreas cultivadas teriam produtividade significativa- 
Tabela 2. Resultados das regressões: Variação de índice de produtividade agregado em relação à variação de área plantada do Brasil de 1995 a 2008, somente para estados de fronteira agrícola

\begin{tabular}{|c|c|c|c|c|}
\hline \multirow{2}{*}{$\begin{array}{l}\text { Coeficiente } \\
\text { (Estatística t) }\end{array}$} & \multicolumn{2}{|c|}{ Modelo B } & \multicolumn{2}{|c|}{ Modelo A } \\
\hline & (1) & (2) & (3) & (4) \\
\hline Variação da área $\left(\beta_{1}\right.$ ou $\left.\delta_{1}\right)$ & $\begin{array}{c}-0,011 \\
(-0,193) \\
\end{array}$ & & $\begin{array}{c}0,143^{* * *} \\
(3,497) \\
\end{array}$ & \\
\hline Variação positiva da área $\left(\beta_{2}\right.$ ou $\left.\delta_{2}\right)$ & & $\begin{array}{c}-0,139 \\
(-1,561) \\
\end{array}$ & & $\begin{array}{c}0,004 \\
(0,054) \\
\end{array}$ \\
\hline Variação negativa da área $\left(\beta_{3}\right.$ ou $\left.\delta_{3}\right)$ & & $\begin{array}{c}0,297 \\
(1,311) \\
\end{array}$ & & $\begin{array}{c}0,267^{* * *} \\
(3,978) \\
\end{array}$ \\
\hline Constante & $\begin{array}{l}-0,073^{*} \\
(-1,964) \\
\end{array}$ & $\begin{array}{c}0,006 \\
(0,082) \\
\end{array}$ & $\begin{array}{c}-0,039 \\
(-0,890) \\
\end{array}$ & $\begin{array}{r}0,014 \\
(0,264) \\
\end{array}$ \\
\hline $\mathrm{R}^{2}$ & 0,202 & 0,210 & 0,265 & 0,284 \\
\hline Observações (N x T) & 169 & 169 & 169 & 169 \\
\hline
\end{tabular}

Notas: Estatística $t$ entre parênteses; *** Significativo a 1\%; ** Significativo a 5\%; * Significativo a 10\%. Dummies de ano incluídas nas estimações. Fonte: Resultados da pesquisa.

mente menor que as áreas já exploradas. Ainda conforme os resultados apresentados nas colunas 2 e 4, nota-se que os parâmetros associados à redução na área cultivada (iguais a 0,28 no caso do Modelo B e 0,31 no Modelo A) são estatisticamente significantes, sugerindo, em ambas as estimações, redução na produtividade conforme ocorrem quedas na área ${ }^{21}$ - de acordo com as estimações, parece ser este o efeito que explica os resultados encontrados nas colunas 1 e 3 desta mesma tabela.

Com vistas a aprofundar as discussões, buscou-se realizar duas extensões da análise: a primeira delas considera as mesmas estimações apresentadas na Tabela 1, mas utilizando apenas os dados dos estados caracterizados como fronteiras agrícolas ${ }^{22}$. Estas estimações foram realizadas visando verificar se há um padrão diferenciado para essas regiões, que são as que apresentam em maior intensidade o fenômeno de expansão das áreas de culturas.

Os resultados referentes à primeira extensão são apresentados na Tabela 2, os quais sugerem um padrão de resultados bastante semelhante

21. Apesar de os parâmetros serem positivos, a variação da área, nestes casos, é sempre negativa, implicando em queda da produtividade.

22. Os estados considerados como de fronteira agrícola foram: Rondônia, Acre, Amazonas, Roraima, Pará, Amapá, Tocantins, Maranhão, Piauí, Bahia, Mato Grosso do Sul, Mato Grosso e Goiás. ao observado na Tabela 1. No caso da coluna 1, a variação da área cultivada continua apresentando um coeficiente estatisticamente nulo e mesmo a decomposição dos efeitos de aumentos e reduções de área continuam gerando resultados sem significância estatística - conforme visto anteriormente, na Tabela 1, o parâmetro referente à redução da área era estatisticamente diferente de zero. Já no caso do Modelo A, a coluna 3 continua indicando, a exemplo da mesma coluna da Tabela 1, que variações da área levam a um aumento do índice de produtividade agrícola (parâmetro igual a 0,14$)$, e quando as variações são tratadas separadamente, continua sendo constatado que apenas as variações negativas da área levam à redução da produtividade - neste caso, o parâmetro obtido indica que uma queda de um ponto percentual na área cultivada reduz a produtividade em 0,26 pontos percentuais, valor este também relativamente semelhante ao observado na coluna 4 da Tabela 1. Desta forma, mesmo nas unidades da federação que fazem parte da chamada fronteira agrícola, os resultados continuam refutando a premissa da legislação californiana.

Como segunda extensão do estudo, procurou-se estimar as mesmas regressões para quatro culturas isoladamente: cana-de-açúcar, soja, milho e arroz, culturas comumente presentes em áreas agrícolas novas ou associadas à conversão de pastagens para culturas. Considerou-se nos 
modelos econométricos a variável dependente como a variação da produtividade individual de cada uma destas culturas e como variável explicativa a expansão da área de culturas total em cada unidade da federação ${ }^{23}$. No caso do Modelo A, utilizou-se a mesma formulação de Bruce e Carriquiry (2010), em que a variação na produtividade absoluta da cultura específica é a variável explicada e a variação na área total de culturas é a variável explicativa. Já na formulação $\mathrm{B}$, consideram-se as variações percentuais anuais tanto na produtividade da cultura quanto na área.

A Tabela 3 apresenta os resultados obtidos para as culturas específicas selecionadas. Os resultados para a cultura da soja revelam parâmetros não significativos mesmo ao nível de 10\%, qualquer que seja a especificação ou modelo considerado. Desta forma, estes resultados sugerem que variações na área cultivada não implicam em alteração na produtividade desta cultura.

Para a cultura do milho, tem-se um padrão de resultados semelhante ao observado na Tabela 1. De acordo com as colunas 5 e 7, os parâmetros indicam que variações na área cultivada levam a aumento na produtividade desta cultura. Contudo, quando as variações positivas e negativas da área são tratadas separadamente (colunas 6 e 8), nota-se que os coeficientes associados a aumentos da área são sempre estatisticamente nulos, ao passo que os coeficientes referentes à

23. É importante ressaltar algumas limitações desta formulação. A relação entre a produtividade de uma dada cultura e a variação da área de todas as culturas pode mascarar que a cultura em questão está se expandindo sobre áreas anteriormente ocupadas com culturas. Portanto, essa formulação não captaria o efeito sobre a produtividade daquela cultura sendo plantada sobre as áreas recentemente convertidas a partir de pastagens ou de vegetação natural. A escolha específica das culturas da soja, cana-de-açúcar, milho e arroz busca minimizar estes efeitos, uma vez que essas culturas são comumente encontradas em áreas que foram convertidas de vegetação natural para agricultura (principalmente no caso da soja) e de pastagens para cultivo agrícola (principalmente no caso da soja e da cana-de-açúcar). Por outro lado, não seria apropriado utilizar como variável explicativa a expansão da área da própria cultura, uma vez que a hipótese sendo testada diz respeito ao efeito da expansão da área agrícola total sobre a produtividade. queda da área cultivada são estatisticamente significantes a $1 \%$, indicando em particular reduções na produtividade desta cultura - no caso do Modelo A, tem-se uma redução de aproximadamente $181 \mathrm{~kg} / \mathrm{ha}$ (que corresponde à aproximadamente $8,4 \%$ da produtividade média desta cultura) quando a área cultivada se reduz em 1 ponto percentual.

No caso do arroz, as colunas 9 e 11 indicam aumento da produtividade quando ocorrem expansões da área cultivada - contudo, somente na segunda destas colunas há coeficientes estatisticamente significantes aos níveis usualmente aceitos. Ao separarem-se os efeitos decorrentes de aumentos e reduções da área, os resultados do Modelo B indicam que expansões levam a aumentos na produtividade (coeficiente igual a 0,14, cuja interpretação vai em direção oposta à assumida pela legislação californiana), embora o coeficiente associado à queda da área seja estatisticamente nulo. Já no caso do Modelo A, tem-se um padrão diferente de resultados: embora o parâmetro associado ao crescimento da área não seja estatisticamente significante, o parâmetro referente à redução da área é estatisticamente não nulo e, em particular, indica que reduções da área levam à redução da produtividade, em linha com os resultados obtidos na Tabela 1 .

Finalmente, para a produção de cana-de-açúcar, nota-se que os parâmetros não são significantes aos níveis usualmente aceitos, qualquer que seja a especificação ou modelo considerado indicando, a exemplo da cultura de soja, que sua produtividade parece não ser alterada por variações na área cultivada.

Em termos gerais, estes resultados, obtidos para algumas das principais culturas anuais do País, apesar de não serem homogêneos entre si em termos de direção e significância dos parâmetros e apesar de não serem totalmente semelhantes aos resultados referentes ao índice geral de produtividade, continuam não confirmando a hipótese assumida pelo relatório CARB, qual seja, de forte queda da produtividade quando da expansão da área agrícola no caso do Brasil. 


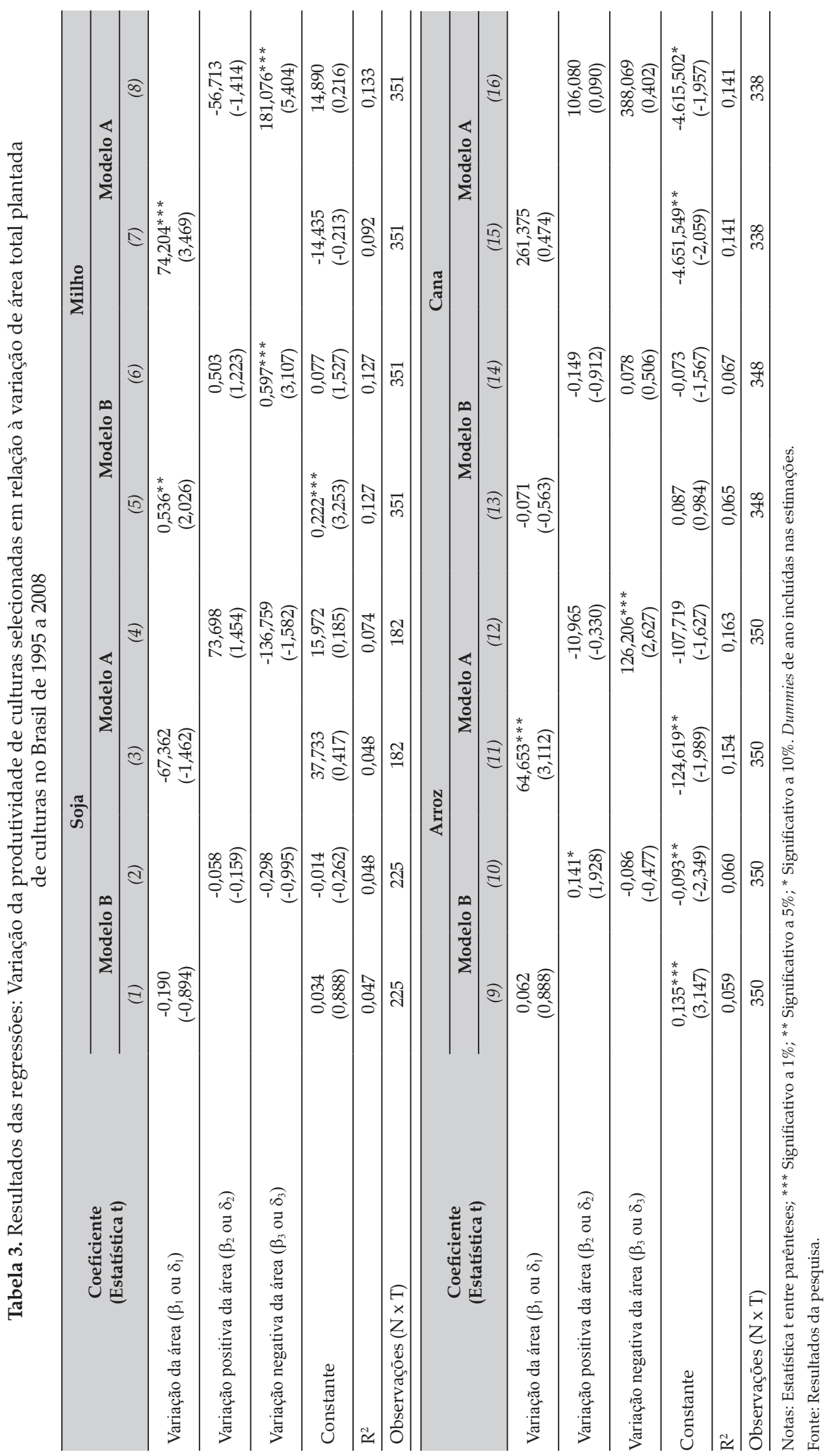




\section{Conclusões}

O presente trabalho procurou contribuir para o debate sobre o papel do etanol de cana-de-açúcar no atual cenário de busca por uma nova matriz energética mundial, questionando e recalculando valores de efeitos indiretos de mudança de uso da terra obtidos e utilizados em uma recente proposta de regulação californiana, chamada aqui de relatório CARB. Em particular, procurou-se estimar se e em que medida a expansão das áreas de culturas afeta a produtividade agrícola no Brasil. Dessa forma, pôde-se testar se, diante de um aumento na área total de culturas promovido pela expansão dos biocombustíveis, haveria queda da produtividade média das culturas no País a ponto de gerar conversão de maiores extensões de áreas de vegetação natural para culturas e, com isso, maiores emissões indiretas de gases de efeito estufa.

Para atingir este objetivo, este estudo considerou um modelo econométrico, baseado na aplicação do método proposto por Bruce e Carriquiry (2010), ampliando-o de forma a considerar um índice de produtividade agrícola agregado e a adição de uma variável dummy que capta o comportamento diferenciado da produtividade diante de expansões ou contrações da área de cultivo de cada estado. Além disso, foi efetuada uma análise alternativa ao modelo de Bruce e Carriquiry (2010), buscando-se investigar a relação de curto prazo entre mudança na área e produtividade.

Os resultados das regressões sugerem que a expansão da área de culturas sobre áreas de pastagens e florestas no Brasil não está relacionada a uma queda de produtividade nessas novas áreas no médio e longo prazo, enquanto para variações anuais observam-se efeitos praticamente nulos. Desta forma, os resultados obtidos no presente estudo sugerem não ser adequado considerar para o Brasil que a conversão de áreas de pastagens e florestas em áreas de culturas seja capaz de reduzir a produtividade destas áreas em $50 \%$ em relação às áreas de cultivo já estabelecidas, como adotado no relatório CARB.
É importante destacar que os resultados encontrados neste estudo podem alterar substancialmente as estimativas do relatório CARB a respeito dos impactos indiretos que a expansão do etanol brasileiro teria sobre as emissões de gases de efeito estufa. Sob a hipótese utilizada naquele relatório, cada hectare a mais de cana-de-açúcar que fosse plantado para produção de etanol geraria a necessidade de conversão de dois hectares de florestas para acomodar o incremento do etanol sem alterar a produção da agropecuária como um todo. Os resultados do presente estudo sugerem que essa relação está equivocada, sendo tal necessidade de conversão de área bem inferior, ao menos de 1:1. As emissões indiretas de gases de efeito estufa, portanto, estão bastante superestimadas no relatório CARB. Tal resultado deve ser considerado pelos agentes do setor privado, entidades de classe e poder público brasileiros nas discussões internacionais sobre os benefícios dos biocombustíveis e possíveis barreiras técnicas no seu comércio internacional.

Vale ainda ressaltar que os resultados obtidos são úteis não apenas no que diz respeito à modelagem de possíveis impactos indiretos no uso da terra a partir do aumento de produção dos biocombustíveis, mas também contribuem para evidenciar que a expansão da agricultura brasileira nas últimas décadas tem sido realizada mantendo-se, ou mesmo elevando-se, o nível tecnológico e de produtividade observado nas áreas agrícolas tradicionais. Isso significa que o agricultor responsável pela conversão de áreas de pastagens ou de florestas em áreas de culturas tem adotado práticas modernas e economicamente sustentáveis, capazes de manter o rendimento por área médio do País, em contraste com os métodos de agricultura de baixa capacidade de suporte baseadas no abandono de áreas exploradas e contínuo avanço sobre áreas de vegetação natural.

Ainda, dada a diversidade das condições de produção e de mercados agrícolas no País, torna-se mais apropriada a adoção de valores de razão de produtividades entre áreas novas e tradicionais regionalmente especificados. Devido à sua 
extensão territorial, seria difícil encontrar um valor único de razão de produtividades para o Brasil. Neste sentido, os resultados sugerem a necessidade de se utilizar valores mais realistas para os parâmetros utilizados nos exercícios de modelagem que fundamentam a legislação californiana, com o objetivo de se alcançar resultados condizentes com a dinâmica dos mercados agrícolas e de energia, entre outros. Por fim, evidencia-se a relevância da pesquisa contínua e o aprimoramento das hipóteses e parâmetros utilizados em modelos econômicos que são utilizados com fins de formulação de políticas ambientais. Futuros trabalhos devem ser desenvolvidos de forma a refinar a hipótese utilizada no Relatório CARB sobre a queda na produtividade de novas áreas agrícolas diante da expansão dos biocombustíveis.

\section{Referências bibliográficas}

AHMED, S. A., HERTEL, T. e LUBOWSKI, R. Calibration of a Land Cover Supply Function Using Transition Probabilities. GTAP Research Memorandum, Center for Global Trade Analysis. Purdue University, West Lafayette, IN, 2008. Disponível em: < http://www. gtap.agecon.purdue.edu/resources/res_display. asp?RecordID=2947>. Acesso em: 18 jul. 2011.

ALBUQUERQUE, A. C. S. e SILVA, G. A. (Eds.). Agricultura tropical - quatro décadas de inovações tecnológicas, institucionais e políticas. Brasília: EMBRAPA, 2008. 1337 p.

ALVES, E. R. A., SOUZA, G. S. e GOMES, E. G. (Eds.). Contribuição da Embrapa para o desenvolvimento da agricultura no Brasil. Brasília: EMBRAPA, 2013. 291 p.

BIRUR, D. K., HERTEL, T. W. e TYNER, W. E. Impact of Biofuel Production on World Agricultural Markets: A Computable General Equilibrium Analysis. Center for Global Trade Analysis. Purdue, 2008. GTAP Working Paper No. 53.

BRUCE, A. B. e CARRIQUIRY, M. An exploration of certain aspects of CARB's approach to modeling indirect land use from expanded biodiesel production. CARD Staff Report, Iowa, Feb. 2010. Disponível em: <www. cardiastate.edu>. Acesso em: 14 jun. 2011.

California Air Resource Board (CARB). Proposed Regulation to Implement the Low Carbon Fuel Standard,
Volume I, mar. 5, 2009. Disponível em: <http://www. arb.ca.gov/fuels/lcfs/030409lcfs_isor_vol1.pdf $>$. Acesso em: 17 ago. 2011.

DIMARANAN, B. V. (Ed.). Global Trade, Assistance, and Production: The GTAP 6 Data Base, Center for Global Trade Analysis, Purdue University, 2006.

EPA. Renewable Fuel Standard (RFS2): Program Amendments; Washington DC, 2010. Disponível em: <http://www.epa.gov/otaq/fuels/renewablefuels/ regulations.htm >. Acesso em: 3 ago. 2011.

EUROPEAN COMMISSION. Impact Assessment of the Renewable Energy Roadmap - March 2007, Directorate-General for Agriculture and Rural Development, European Commission, AGRI G-2/ WM D, 2007). Disponível em: <http://ec.europa.eu/ agriculture-/analysis/markets/biofuel/impact042007/ text_en.pdf $>$. Acesso em: 3 mar. 2011.

FARGIONE, J., HILL, J., TILMAN, D., POLASKY, S. e HAWTHORNE, P. Land clearing and the biofuel carbon debt. Science, v. 319, p. 1235-1238, 2008.

GASQUES, J. G., BASTOS, E. T., BACCHI, M. R. P. e VALDES, C. Produtividade total dos fatores e transformações da agricultura brasileira: análise dos dados dos censos agropecuários. In: GASQUES, J. G., VIEIRA FILHO, J. E. R. e NAVARRO, Z. (Eds.). A Agricultura Brasileira: desempenho, desafios e perspectivas, Brasilia: IPEA, 2010, p. 19-44.

HERTEL, T. W. Global Trade analysis: modeling and applications. New York: Cambridge University Press, 1997.

HUANG, H. e KHANNA, M. An econometric analysis of U.S. crop yield and cropland acreage: implications for the impact of climate change. In: The Agricultural \& Applied Economics Association, 2010. AAEA, CAES, \& WAEA Joint Annual Meeting, Denver, Colorado, July 25-27, 2010. Disponível em: < http://ageconsearch. umn.edu/handle/61527> . Acesso em: 3 mar. 2011.

LEE, H., HERTEL, T. W., SOHNGEN, B. e RAMANKUTTY, N. Towards an Integrated Land Use Data Base for Assessing the Potential for Greenhouse Gas Mitigation. GTAP Technical Paper 25, Global Trade Analysis Project, West Lafayette, 2005. Disponível em: <https://www.gtap.agecon.purdue.edu/resources/ download/2375.pdf>. Acesso em: 25 ago. 2011.

LUBOWSKI, R. N., PLANTINGA, A. J. e STAVINS, R. $\mathrm{N}$. Land-use change and carbon sinks: econometric estimation of the carbon sequestration supply function. Journal of Environmental Economics and Management, v. 51 , p. $135-152,2006$. 
MARTHA JR., G. B., ALVES. E. e CONTINI, E. Landsaving approaches and beef production growth in Brazil. Agricultural Systems, v. 110, p. 173-177, 2012.

MELILLO, J. M., REILLY, J. M., KICKLIGHTER, D. W., GURGEL, A. C., CRONIN, T. W., PALTSEV, S., FELZER, B. S., WANG, X., SOKOLOV, A. P. e SCHLOSSER, C. A. Indirect Emissions from Biofuels: How Important? Science, v. 326, p. 1397-1399, 2009.

ROBERTS, M. J. e SCHLENKER, W. Identifying supply and demand elasticities of agricultural commodities: implications for the US ethanol mandate. NBER Working Paper 15921, Cambridge MA, April 2010.

SEARCHINGER, T., HEIMLICH, R., HOUGHTON, R. A., DONG, F., ELOBEID, A., FABIOSA, J., TOKGOZ, S., HAYES, D. e YU, T. Use of U.S. croplands for biofuels increases greenhouse gases through emissions from land-use change. Science, v. 319, p. 1238-1240, 2008.

TAHERIPOUR, F., BIRUR, D. K., HERTEL, T. W. e TYNER, W. E. Introducing liquid biofuels into the GTAP data base. Center for Global Trade Analysis, Purdue, 2007. Research Memorandum 11.

TYNER, W. E., TAHERIPOUR, F., ZHUANG, Q., BIRUR, D. e BALDOS, U. Land Use Changes and Consequent CO2 Emissions due to US Corn Ethanol Production: A Comprehensive Analysis. Working paper. Purdue University, West Lafayette, IN. 2010. Disponível em: $<$ http://web.ics.purdue.edu/ qzhuang/Dr_\%20 Qianlai\%20Zhuang_files/Argonne-GTAP_2010_ With\%20Appendices\%20and\%20exec\%20sum4.pdf>. Acesso em: 5 jul. 2011.

ZHUANG, Q., MCGUIRE, A. D., MELILLO, J. M., CLEIN, J. S., DARGAVILLE, R. J., KICKLIGHTER, D. W., MYNENI, R. B., DONG, J., ROMANOVSKY, V. E., HARDEN, J. e HOBBIE, J. E. Carbon cycling in extratropical terrestrial ecosystems of the Northern Hemisphere during the 20th Century: a modeling analysis of the influences of soil thermal dynamics. Tellus, v. 55, n. B, 2003. 